\title{
Stock Market, Economic Performance, And Presidential Elections
}

Wen-Wen Chien, State University of New York at Old Westbury, USA

Roger W. Mayer, Walden University, USA

Zigan Wang, Columbia University, USA

\begin{abstract}
Using stock market and economic data from 1900 to 2008 from 27 separate presidential administrations in the United States (U.S.), including 15 Republican and 12 Democratic, this paper examines the relationships between the market return after each Election Day and economic performance during the presidential term. Using the theoretical framework of political economy, the authors examine how Wall Street's reaction to a presidential election acts as a predictive measure of future economic performance. The analysis shows that the after-election market movement has progressively been more accurate in predicting the future Gross Domestic Product $(G D P)$ growth but not the future unemployment rates. Given that the results show a higher correlation over time, the model appears to provide a good starting point for judging the economic potential of future presidential administrations.
\end{abstract}

Keywords: Market Return; Economic Performance; GDP Growth; Unemployment

\section{INTRODUCTION}

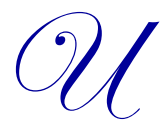

S. presidential elections and the stock market are popular topics for research (Wisniewski, Lightfoot, \& Lilley, 2012). The reason for this interest is because policies established by the governing president affect the ability of businesses and the general economy to prosper. The political economy theoretical framework provides a basis for understanding the relationship between politics and the economy. Gilpin (2001) describes this relationship as "interactive." As is seen from the prism of historic events, business attempts to promote a political agenda that supports their goals (Caro, 2002). The reason that some business sectors are willing to spare massive amounts of money to promote a specific candidate or political agenda is because the winning candidate's agenda has a direct impact on the business environment. Allvine and O'Neil (1980) documented the interaction between politics and the market by demonstrating that markets generally follow a four-year business cycle that corresponds to the presidential election cycle. The authors' research adds to this perspective by increasing their understanding of how well business and investors gauge the final decision of a presidential election.

Prior researchers examined various elements of the election process to understand the relationship between the presidential performance variables and stock market performance. These studies are generally predictive in nature. Niederhoffer, Gibbs, and Bullock (1970) documented the changes of Dow Jones Industrial Average (DJI) before and after election and nominating conventions for 18 presidents from 1900 to 1968 . They also document the one-day, one-week and one-month DJI changes after the events and DJI changes during each of the four years under each president's administration, reaching a conclusion that the stock market performances during Republican and Democratic administrations have no systematic difference. Using data between 1927 and 1998, Santa-Clara and Valkanov (2003) determined that the stock market's excess return is higher under Democratic than Republican presidencies and the difference is from higher real stock returns and lower real interest rates but is not explained by business-cycle variables and is not concentrated around election dates.

Goodell and Vähämaa (2013) demonstrated that the presidential election process creates market uncertainty as investors develop expectations regarding potential winners and future macroeconomic policy. Goodell and Bodey (2012) determined that as the probable winner of the election becomes clearer, volatility decreases and markets react 
negatively with decreases in P/E ratios. Riley and Luksetich (1980) suggest that the results are dependent upon what party becomes the clear winner. Huang (1985) documents the higher average returns during Democratic administrations, in contrast of the widely held belief that the Republican Party is better at business. Moreover, findings from Johnson, Chittenden, and Jensen (1999) also indicate that the returns to small-cap stocks are substantially higher during Democratic administrations.

The analysis shows that the after-election market movement has progressively been more accurate in predicting the future GDP growth but not the future unemployment rates. Given that the researchers see a higher correlation over time, their model appears to provide a good starting point for judging the economic potential of new presidential administrations. An additional finding that was not part of the hypothesis was that Republicans tend to govern in an economy with unemployment rates that increase during the course of their term. Democrats tend to preside over an economy with higher unemployment rates at the beginning of their terms and that tend to decrease over time.

The reminder of this paper consists of the research question and hypotheses, research model and data used to test hypotheses, empirical results, and a conclusion.

\section{RESEARCH QUESTION AND HYPOTHSES}

The overreaching research question for this study is, "to what extent does the stock price change immediately after a presidential election relate to or predict the presidential administration' economic performance as defined by GDP growth and unemployment?"

H1: There is a relationship between the GDP growth during the term of a presidential administration and the change in stock price immediately after the presidential election.

H2: There is a relationship between unemployment rates during the term of a presidential administration and the change in stock price immediately after the presidential election.

Instead of confining their research to just market reactions to the new president, the researchers examined the relationship between the market's perspective of each president and the economic performance under each one, including GDP growth and the unemployment rate. The goal of this research is to determine if the market is able to predict the impact of a president on the economy. The hypotheses are based on the assumption that the stock market movement reflects people's perspective of the future economy. The generalized assumption is in the statement that when people are positive about the future, the stock market reflects this attitude and increases. Conversely, when people have a pessimistic view of the future the market reflects this attitude and decreases. The efficient market hypothesis suggests that the market responds to the election winners with all the available information about the winning candidate. Thus, the prediction of an efficient market should be right (Fama, 1970). If the market moves up after the election, it suggests that the market generally approves the winner's capability of making the economy grow; if the market drops, it suggests that the investors are pessimistic about the economy in the future. To the authors' knowledge, there has not been any research verifying the accuracy of the market's prediction and this paper will fill in this gap.

\section{RESEARCH MODEL}

To test the hypothesis, the researchers use the simple OLS linear regression model to address the correlation between the 1-day DJI change following the Election Day and the economic indicators, the four-year cumulative GDP growth rate and average unemployment rate. The formula of the model is:

$$
E I_{t}=\beta_{0}+\beta_{1} D J I_{t}+\beta_{2} X_{t}+\varepsilon_{t}
$$

where $E I$ is the specific economic indicator. The researchers used two separate indicators, including the four-year cumulative GDP growth rate in regressions (1) - (4) and the average unemployment rate in regressions (5) - (6). DJI is the 1-day DJI percentage change following the Election Day. $X$ is the other explanatory variable, or the four-year lag 
cumulative GDP growth rate in regressions (1) and (3). The lag GDP growth is a common independent variable for explaining or predicting the GDP growth rate.

\section{DATA}

The DJI was used to measure the market movement around the Election Day because it has a longer range than any other market indices and it traces back to before 1900. The data include stock market and economic information from 1900 to 2008 across 27 administrations with 15 of them Republican and 12 Democratic. Table 1 summarizes the DJI closing price of selected dates in each election year's November and December around the Election Day since 1900. All data is publicly available; DJI was extracted from Bloomberg. ${ }^{1}$ Some data reported by Bloomberg were different compared to the dataset used by Niederhoffer et al. (1970). The researchers were unable to determine the source of this error; however, the differences did not affect the results of hypothesis testing. In 1984, the NYSE opened on Election Day for the first time in its 192-year history. So, starting in 1984, the data used was the Election Day (Tuesday) closing price rather than the price on Monday before Election Day.

Table 1 (as well as Table 3) shows that among the 13 times that the Democratic Party won the elections, the DJI dropped nine times after the Election Day and among the 15 times when the Republicans won, the market rose ten times during Wednesday. The sample does not include Obama's victory in 2012, but the DJI dropped on Wednesday after the election. Therefore, since 1900, the market has responded negatively to the Democratic Party's winning for 10 out of 14 times in total.

A correlation analysis concludes that the DJI change on Wednesday is closely correlated to the DJI movement within the election week (Friday). In addition, it correlates with the DJI movement within two weeks (the next Friday) and the DJI return within a month after the election. For brevity, the researchers did not include this analysis in the tables.

Table 2 summarizes the yearly GDP growth and unemployment rates since 1900. GDP growth data from 1901 to 1996 are from Angus Maddison's (2008) Historical Statistics of the World Economy: 1-2008 AD. GDP growth data from 1997 to 2011 are from the World Bank. GDP growth in 2012 is from the U.S. Bureau of Economic Analysis. The researchers obtained unemployment rates from various sources. Unemployment rates from 1901 to 1930 are from Romer (1986), data from 1931 to 1940 are from Darby (1976), data from 1941 to 1947 are from Barro (1977), and data after 1948 are from the U.S. Bureau of Labor Statistics. The data used for this study comes from the "age 16 and over" category (U.S. Bureau of Labor Statistics, 2013). According to Barro (1977), the annual average unemployment rates (data are given in the Economic Report of the President) are based on the total labor force, which includes military personnel. Data for 1941-43 is adjusted for treatment of government "emergency workers," as discussed by Darby (1976). Cumulative GDP growth and average unemployment rate are calculated according to each year's data.

There were some winning candidates who did not finish their four-year presidency for various reasons. In recent times, this includes John F. Kennedy who was assassinated and Richard Nixon who resigned in his second term. In each case, the Vice-President took over and completed the terms. Therefore, the economic performance of those years should be attributed to combined administration rather than to any single president.

\footnotetext{
${ }^{1}$ http://www.bloomberg.com/markets/stocks/
} 
Table 1: Summary of Dow Jones Industrial Average (DJI) Around Election Day*

\begin{tabular}{|c|c|c|c|c|c|c|c|c|c|c|c|c|c|c|}
\hline$\dot{\bar{J}}$ & 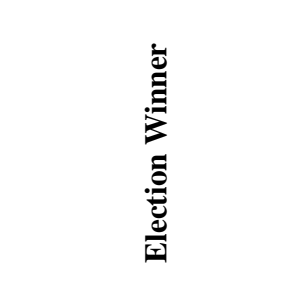 & : & 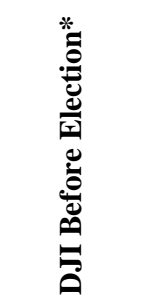 & 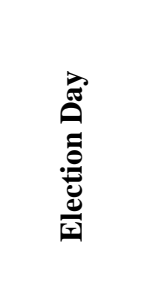 & 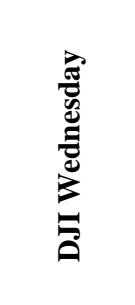 & 兽 & 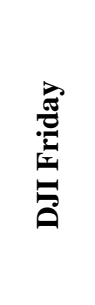 & 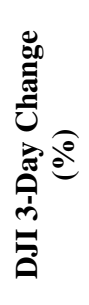 & 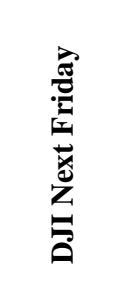 & 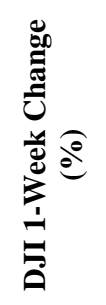 & 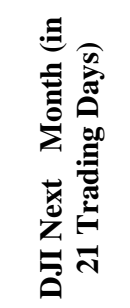 & 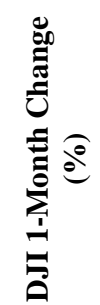 & 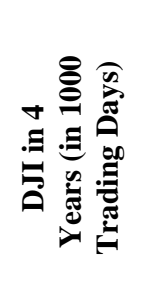 & 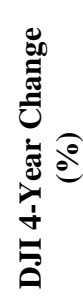 \\
\hline 190 & William McKinley & $(\mathrm{R})$ & 60.87 & $11 / 6 / 190$ & 62.9 & 3.33 & 65.15 & 7.03 & 68.19 & 12.03 & 65.07 & 6.90 & 63.72 & 4.68 \\
\hline 190 & Theodore Roosevelt & $(\mathrm{R})$ & 66.21 & $11 / 8 / 190$ & 67.07 & 1.30 & 68.03 & 2.75 & 69.69 & 5.26 & 68 & 2.70 & 82.22 & 24.18 \\
\hline 190 & William Howard Taft & $(\mathrm{R})$ & 82.9 & $11 / 3 / 190$ & 84.87 & 2.38 & 87.28 & 5.28 & 88.38 & 6.61 & 86.58 & 4.44 & 91.44 & 10.30 \\
\hline 191 & Woodrow Wilson & (D) & 90.29 & $11 / 5 / 191$ & 91.94 & 1.83 & 91.31 & 1.13 & 90.09 & -0.22 & 87.88 & -2.67 & 95.05 & 5.27 \\
\hline 191 & Woodrow Wilson & (D) & 107.21 & $11 / 7 / 191$ & 106.83 & -0.35 & 107.7 & 0.41 & 109.62 & 2.25 & 106.43 & -0.73 & 76.65 & -28.5 \\
\hline 192 & Warren G. Harding & (R) & 85.48 & $11 / 2 / 192$ & 84.99 & -0.57 & 83.48 & -2.34 & 77.56 & -9.27 & 77.3 & -9.57 & 101.96 & 19.28 \\
\hline 192 & Calvin Coolidge & (R) & 103.89 & $11 / 4 / 192$ & 105.11 & 1.17 & 104.9 & 0.93 & 108.96 & 4.88 & 111.56 & 7.38 & 257.13 & 147.5 \\
\hline 192 & Herbert Hoover & (R) & 257.58 & $11 / 6 / 192$ & 260.68 & 1.20 & 263.1 & 2.12 & 276.66 & 7.41 & 279.79 & 8.62 & 61.86 & -75.9 \\
\hline 193 & Franklin D. Roosevelt & (D) & 64.58 & $11 / 8 / 193$ & 61.67 & -4.51 & 68.03 & 5.34 & 62.96 & -2.51 & 60.05 & -7.01 & 182.25 & 182.2 \\
\hline 193 & Franklin D. Roosevelt & (D) & 176.67 & $11 / 3 / 193$ & 180.66 & 2.26 & 181.6 & 2.79 & 182.65 & 3.38 & 180.97 & 2.43 & 132.45 & -25.0 \\
\hline 194 & Franklin D. Roosevelt & (D) & 135.21 & $11 / 5 / 194$ & 131.98 & -2.39 & 136.6 & 1.06 & 134.74 & -0.35 & 130.33 & -3.61 & 148.87 & 10.10 \\
\hline 194 & Franklin D. Roosevelt & (D) & 147.92 & $11 / 7 / 194$ & 147.52 & -0.27 & 148.1 & 0.11 & 145.77 & -1.45 & 149.23 & 0.89 & 188.28 & 27.29 \\
\hline 194 & Harry S Truman & (D) & 189.76 & $11 / 2 / 194$ & 182.46 & -3.85 & 178.4 & -6.00 & 176.01 & -7.25 & 175 & -7.78 & 265.83 & 40.09 \\
\hline 195 & Dwight D. Eisenhower & (R) & 270.22 & $11 / 4 / 195$ & 271.29 & 0.40 & 273.5 & 1.20 & 274.44 & 1.56 & 282.05 & 4.38 & 490.18 & 81.40 \\
\hline 195 & Dwight D. Eisenhower & $(\mathrm{R})$ & 495.36 & $11 / 6 / 195$ & 491.14 & -0.85 & 485.3 & -2.02 & 480.66 & -2.97 & 492.73 & -0.53 & 587.30 & 18.56 \\
\hline 196 & John F. Kennedy & (D) & 597.62 & $11 / 8 / 196$ & 602.25 & 0.77 & 608.6 & 1.84 & 603.61 & 1.00 & 605.16 & 1.26 & 876.20 & 46.61 \\
\hline 196 & Lyndon Johnson & (D) & 875.5 & $11 / 3 / 196$ & 873.81 & -0.19 & 876.9 & 0.16 & 874.1 & -0.16 & 870.78 & -0.54 & 950.65 & 8.58 \\
\hline 196 & Richard Nixon & $(\mathrm{R})$ & 946.23 & $11 / 5 / 196$ & 949.47 & 0.34 & 959 & 1.35 & 963.7 & 1.85 & 977.69 & 3.32 & 930.46 & -1.67 \\
\hline 197 & Richard Nixon & (R) & 984.8 & $11 / 7 / 197$ & 983.74 & -0.11 & 995.3 & 1.06 & 1005.57 & 2.11 & 1033.26 & 4.92 & 937.00 & -4.85 \\
\hline 197 & Jimmy Carter & (D) & 966.09 & $11 / 2 / 197$ & 956.53 & -0.99 & 943.1 & -2.38 & 927.69 & -3.97 & 946.64 & -2.01 & 959.90 & -0.64 \\
\hline 198 & Ronald Reagan & $(\mathrm{R})$ & 937.2 & $11 / 4 / 198$ & 953.16 & 1.70 & 932.4 & -0.51 & 986.35 & 5.24 & 970.48 & 3.55 & 1195.89 & 27.60 \\
\hline 198 & Ronald Reagan & $(\mathrm{R})$ & 1229.24 & $11 / 6 / 198$ & 1233.22 & 0.32 & 1219 & -0.84 & 1187.94 & -3.36 & 1170.49 & -4.78 & 2183.50 & 77.63 \\
\hline 198 & George Bush & (R) & 2124.64 & $11 / 8 / 198$ & 2118.24 & -0.30 & 2067 & -2.71 & 2062.41 & -2.93 & 2141.71 & 0.80 & 3200.88 & 50.66 \\
\hline 199 & Bill Clinton & (D) & 3262.21 & $11 / 3 / 199$ & 3223.04 & -1.20 & 3240 & -0.68 & 3233.03 & -0.89 & 3276.53 & 0.44 & 6059.19 & 85.74 \\
\hline 199 & Bill Clinton & (D) & 6041.67 & $11 / 5 / 199$ & 6177.71 & 2.25 & 6220 & 2.95 & 6348.03 & 5.07 & 6437.1 & 6.55 & 10271.72 & 70.01 \\
\hline 200 & George W. Bush & (R) & 10977.21 & $11 / 7 / 200$ & 10907.06 & -0.64 & 10603 & -3.41 & 10629.9 & -3.16 & 10617.36 & -3.28 & 10137.05 & -7.65 \\
\hline 200 & George W. Bush & (R) & 10054.39 & $11 / 2 / 200$ & 10137.05 & 0.82 & 10388 & 3.31 & 10539 & 4.82 & 10585.12 & 5.28 & 8519.21 & -15.2 \\
\hline 200 & Barack Obama & (D) & 9625.28 & $11 / 4 / 200$ & 9139.27 & -5.05 & 8944 & -7.08 & 8497.31 & -11.72 & 8635.42 & -10.28 & 13077.34 & 35.86 \\
\hline
\end{tabular}

* DJI closing price of the day just before election results were revealed, which was Monday before 1980 and Tuesday since 1984. 
Table 2: Summary of Yearly GDP Growth and Unemployment Rate under Each President's Administration

\begin{tabular}{|c|c|c|c|c|c|c|c|c|c|c|c|c|c|c|c|}
\hline \multirow[b]{2}{*}{ President } & \multirow[b]{2}{*}{$\stackrel{\vec{E}}{E}$} & \multicolumn{3}{|c|}{ First Year } & \multicolumn{3}{|c|}{ Second Year } & \multicolumn{3}{|c|}{ Third Year } & \multicolumn{3}{|c|}{ Fourth Year } & \multicolumn{2}{|c|}{ All Four Years } \\
\hline & & ప્ & 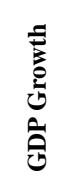 & 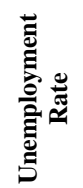 & $\underset{\nu}{ٍ ٍ ~}$ & 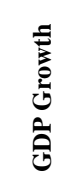 & 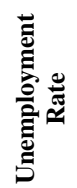 & ঠ્ & 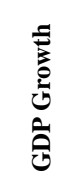 & 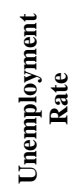 & $\stackrel{\check{\Xi}}{\check{\Xi}}$ & 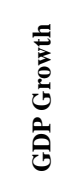 & 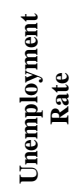 & 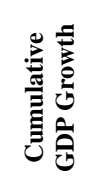 & 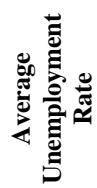 \\
\hline W. McKinley/T. Roosevelt & (R) & 1901 & 11.3 & 4.59 & 1902 & 1.04 & 4.30 & 1903 & 4.86 & 4.35 & 1904 & -1.26 & 5.08 & 16.395 & 4.580 \\
\hline Theodore Roosevelt & (R) & 1905 & 7.40 & 4.62 & 1906 & 11.5 & 3.29 & 1907 & 1.54 & 3.57 & 1908 & -8.19 & 6.17 & 11.657 & 4.413 \\
\hline William Howard Taft & (R) & 1909 & 12.2 & 5.13 & 1910 & 1.02 & 5.86 & 1911 & 3.26 & 6.27 & 1912 & 4.68 & 5.25 & 22.550 & 5.628 \\
\hline Woodrow Wilson & (D) & 1913 & 3.95 & 4.93 & 1914 & $\begin{array}{l}-7.7 \\
\end{array}$ & 6.63 & 1915 & 2.82 & 7.18 & 1916 & 13.80 & 5.63 & 12.265 & 6.093 \\
\hline Woodrow Wilson & (D) & 1917 & -2.50 & 5.23 & 1918 & 9.02 & 3.38 & 1919 & 0.87 & 2.95 & 1920 & -0.95 & 5.16 & 6.201 & 4.180 \\
\hline W. G. Harding/C. Coolidge & (R) & 1921 & -2.27 & 8.73 & 1922 & 5.53 & 6.93 & 1923 & 13.2 & 4.80 & 1924 & 3.06 & 5.80 & 20.310 & 6.565 \\
\hline Calvin Coolidge & (R) & 1925 & 2.32 & 4.92 & 1926 & 6.52 & 4.02 & 1927 & 1.0 & 4.57 & 1928 & 1.12 & 5.02 & 11.314 & 4.633 \\
\hline Herbert Hoover & (R) & 1929 & 6.12 & 4.61 & 1930 & -8.9 & 8.94 & 1931 & -7.8 & 15.3 & 1932 & -13.20 & 22.90 & -22.530 & 12.938 \\
\hline Franklin D. Roosevelt & (D) & 1933 & -2.10 & 20.60 & 1934 & 7.73 & 16.00 & 1935 & 7.65 & 14.20 & 1936 & 14.21 & 9.90 & 29.669 & 15.175 \\
\hline Franklin D. Roosevelt & (D) & 1937 & 4.28 & 9.10 & 1938 & -3.98 & 12.50 & 1939 & 7.96 & 11.30 & 1940 & 7.73 & 9.50 & 16.456 & 10.600 \\
\hline Franklin D. Roosevelt & (D) & 1941 & 18.20 & 5.80 & 1942 & 20.01 & 2.90 & 1943 & 19.89 & 1.50 & 1944 & 8.38 & 1.00 & 84.318 & 2.800 \\
\hline F. D. Roosevelt/ H. Truman & (D) & 1945 & -4.02 & 1.60 & 1946 & -20.64 & 3.70 & 1947 & -1.51 & 3.80 & 1948 & 3.78 & 3.80 & -22.145 & 3.225 \\
\hline Harry S. Truman & (D) & 1949 & 0.39 & 5.90 & 1950 & 8.69 & 5.30 & 1951 & 7.61 & 3.30 & 1952 & 3.73 & 3.00 & 21.797 & 4.375 \\
\hline Dwight D. Eisenhower & (R) & 1953 & 4.60 & 2.90 & 1954 & -0.66 & 5.50 & 1955 & 7.07 & 4.40 & 1956 & 1.95 & 4.10 & 13.426 & 4.225 \\
\hline Dwight D. Eisenhower & (R) & 1957 & 1.88 & 4.30 & 1958 & -1.01 & 6.80 & 1959 & 7.42 & 5.50 & 1960 & 2.49 & 5.50 & 11.032 & 5.525 \\
\hline J. F. Kennedy/L. Johnson & (D) & 1961 & 2.33 & 6.70 & 1962 & 6.03 & 5.50 & 1963 & 4.32 & 5.70 & 1964 & 5.79 & 5.20 & 19.741 & 5.775 \\
\hline Lyndon Johnson & (D) & 1965 & 6.38 & 4.50 & 1966 & 6.55 & 3.80 & 1967 & 2.50 & 3.80 & 1968 & 4.76 & 3.60 & 21.712 & 3.925 \\
\hline Richard Nixon & (R) & 1969 & 3.13 & 3.50 & 1970 & 0.17 & 4.90 & 1971 & 3.12 & 5.90 & 1972 & 5.30 & 5.60 & 12.174 & 4.975 \\
\hline R. Nixon/G. R. Ford & (R) & 1973 & 5.68 & 4.90 & 1974 & -0.28 & 5.60 & 1975 & -0.28 & 8.50 & 1976 & 5.24 & 7.70 & 10.596 & 6.675 \\
\hline Jimmy Carter & (D) & 1977 & 4.53 & 7.10 & 1978 & 5.71 & 6.10 & 1979 & 3.40 & 5.80 & 1980 & 0.05 & 7.10 & 14.313 & 6.525 \\
\hline Ronald Reagan & (R) & 1981 & 2.50 & 7.60 & 1982 & -1.87 & 9.70 & 1983 & 4.19 & 9.60 & 1984 & 7.28 & 7.50 & 12.427 & 8.600 \\
\hline Ronald Reagan & (R) & 1985 & 3.88 & 7.20 & 1986 & 3.44 & 7.00 & 1987 & 3.52 & 6.20 & 1988 & 4.21 & 5.50 & 15.919 & 6.475 \\
\hline George Bush & (R) & 1989 & 3.46 & 5.30 & 1990 & 1.75 & 5.60 & 1991 & -0.19 & 6.80 & 1992 & 3.34 & 7.50 & 8.580 & 6.300 \\
\hline Bill Clinton & (D) & 1993 & 2.69 & 6.90 & 1994 & 4.06 & 6.10 & 1995 & 2.54 & 5.60 & 1996 & 3.75 & 5.40 & 13.682 & 6.000 \\
\hline Bill Clinton & (D) & 1997 & 4.50 & 4.90 & 1998 & 4.40 & 4.50 & 1999 & 4.90 & 4.20 & 2000 & 4.20 & 4.00 & 19.250 & 4.400 \\
\hline George W. Bush & (R) & 2001 & 1.10 & 4.70 & 2002 & 1.80 & 5.80 & 2003 & 2.60 & 6.00 & 2004 & 3.50 & 5.50 & 9.292 & 5.500 \\
\hline George W. Bush & (R) & 2005 & 3.10 & 5.10 & 2006 & 2.70 & 4.60 & 2007 & 1.90 & 4.60 & 2008 & -0.40 & 5.80 & 7.464 & 5.025 \\
\hline Barack Obama & (D) & 2009 & -3.50 & 9.30 & 2010 & 3.00 & 9.60 & 2011 & 1.70 & 9.00 & 2012 & 2.20 & 8.10 & 3.309 & 9.000 \\
\hline Average & & & 3.63 & 6.10 & & 2.34 & 6.24 & & 3.93 & 6.24 & & 3.23 & 6.30 & 14.33 & 6.22 \\
\hline Average since 1953 & & & 3.08 & 5.66 & & 2.39 & 6.07 & & 3.25 & 6.11 & & 3.58 & 5.87 & 12.86 & 5.93 \\
\hline Average (Republican) & & & 4.43 & 5.21 & & 1.52 & 5.92 & & 3.03 & 6.42 & & 1.27 & 6.99 & 10.71 & 6.14 \\
\hline Average since $1953(\mathrm{R})$ & & & 3.26 & 5.06 & & 0.67 & 6.17 & & 3.26 & 6.39 & & 3.66 & 6.08 & 11.21 & 5.92 \\
\hline Average (Democratic) & & & 2.70 & 7.12 & & 3.30 & 6.62 & & 4.97 & 6.03 & & 5.49 & 5.49 & 18.51 & 6.31 \\
\hline Average since 1953 (D) & & & 2.82 & 6.57 & & 4.96 & 5.93 & & 3.23 & 5.68 & & 3.46 & 5.57 & 15.33 & 5.94 \\
\hline
\end{tabular}




\begin{tabular}{|c|c|c|c|c|c|c|c|c|c|c|c|c|}
\hline 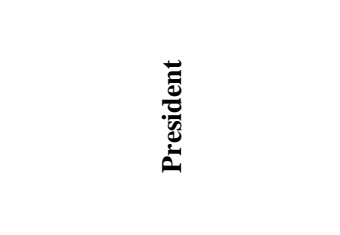 & $\stackrel{\vec{E}}{\vec{E}}$ & $\stackrel{\bar{\Xi}}{\grave{\Xi}}$ & 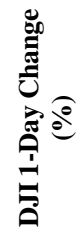 & 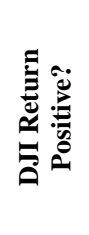 & 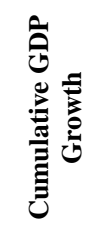 & 总总 & 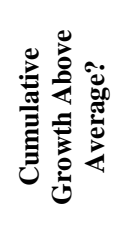 & 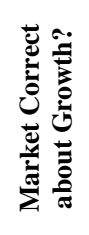 & 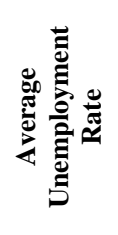 & 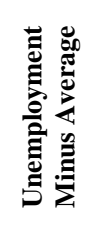 & 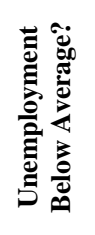 & 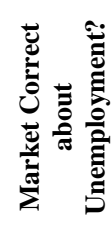 \\
\hline W. McKinley/T. Roosevelt & (R) & 1900 & 3.33 & Yes & 16.395 & 2.065 & Yes & Yes & 4.580 & -1.640 & Yes & Yes \\
\hline Theodore Roosevelt & (R) & 1904 & 1.30 & Yes & 11.657 & -2.673 & No & No & 4.413 & -1.808 & Yes & Yes \\
\hline William Howard Taft & (R) & 1908 & 2.38 & Yes & 22.550 & 8.220 & Yes & Yes & 5.628 & -0.593 & Yes & Yes \\
\hline Woodrow Wilson & (D) & 1912 & 1.83 & Yes & 12.265 & -2.065 & No & No & 6.093 & -0.128 & Yes & Yes \\
\hline Woodrow Wilson & (D) & 1916 & -0.35 & No & 6.201 & -8.129 & No & Yes & 4.180 & -2.040 & Yes & No \\
\hline W. G. Harding/C. Coolidge & $(\mathrm{R})$ & 1920 & -0.57 & No & 20.310 & 5.980 & Yes & No & 6.565 & 0.345 & No & Yes \\
\hline Calvin Coolidge & $(\mathrm{R})$ & 1924 & 1.17 & Yes & 11.314 & -3.016 & No & No & 4.633 & -1.588 & Yes & Yes \\
\hline Herbert Hoover & $(\mathrm{R})$ & 1928 & 1.20 & Yes & -22.530 & -36.860 & No & No & 12.938 & 6.718 & No & No \\
\hline Franklin D. Roosevelt & (D) & 1932 & -4.51 & No & 29.669 & 15.339 & Yes & No & 15.175 & 8.955 & No & Yes \\
\hline Franklin D. Roosevelt & (D) & 1936 & 2.26 & Yes & 16.456 & 2.126 & Yes & Yes & 10.600 & 4.380 & No & No \\
\hline Franklin D. Roosevelt & (D) & 1940 & -2.39 & No & 84.318 & 69.988 & Yes & No & 2.800 & -3.420 & Yes & No \\
\hline F. D. Roosevelt/H. Truman & (D) & 1944 & -0.27 & No & -22.145 & -36.475 & No & Yes & 3.225 & -2.995 & Yes & No \\
\hline Harry S Truman & (D) & 1948 & -3.85 & No & 21.797 & 7.467 & Yes & No & 4.375 & -1.845 & Yes & No \\
\hline Dwight D. Eisenhower & $(\mathrm{R})$ & 1952 & 0.40 & Yes & 13.426 & -0.904 & No & No & 4.225 & -1.995 & Yes & Yes \\
\hline Dwight D. Eisenhower & (R) & 1956 & -0.85 & No & 11.032 & -3.298 & No & Yes & 5.525 & -0.695 & Yes & No \\
\hline John F. Kennedy/L. Johnson & (D) & 1960 & 0.77 & Yes & 19.741 & 5.411 & Yes & Yes & 5.775 & -0.445 & Yes & Yes \\
\hline Lyndon Johnson & (D) & 1964 & -0.19 & No & 21.712 & 7.382 & Yes & No & 3.925 & -2.295 & Yes & No \\
\hline Richard Nixon & $(\mathrm{R})$ & 1968 & 0.34 & Yes & 12.174 & -2.156 & No & No & 4.975 & -1.245 & Yes & Yes \\
\hline R. Nixon/Gerald R. Ford & (R) & 1972 & -0.11 & No & 10.596 & -3.734 & No & Yes & 6.675 & 0.455 & No & Yes \\
\hline Jimmy Carter & (D) & 1976 & -0.99 & No & 14.313 & -0.017 & No & Yes & 6.525 & 0.305 & No & Yes \\
\hline Ronald Reagan & $(\mathrm{R})$ & 1980 & 1.70 & Yes & 12.427 & -1.903 & No & No & 8.600 & 2.380 & No & No \\
\hline Ronald Reagan & (R) & 1984 & 0.32 & Yes & 15.919 & 1.589 & Yes & Yes & 6.475 & 0.255 & No & No \\
\hline George Bush & (R) & 1988 & -0.30 & No & 8.580 & -5.750 & No & Yes & 6.300 & 0.080 & No & Yes \\
\hline Bill Clinton & (D) & 1992 & -1.20 & No & 13.682 & -0.648 & No & Yes & 6.000 & -0.220 & Yes & No \\
\hline Bill Clinton & (D) & 1996 & 2.25 & Yes & 19.250 & 4.920 & Yes & Yes & 4.400 & -1.820 & Yes & No \\
\hline George W. Bush & (R) & 2000 & -0.64 & No & 9.292 & -5.038 & No & Yes & 5.500 & -0.720 & Yes & No \\
\hline George W. Bush & (R) & 2004 & 0.82 & Yes & 7.464 & -6.866 & No & No & 5.025 & -1.195 & Yes & Yes \\
\hline Barack Obama & (D) & 2008 & -5.05 & No & 3.309 & -11.021 & No & Yes & 9.000 & 2.780 & No & Yes \\
\hline
\end{tabular}




\section{EMPIRICAL RESULTS}

\section{Descriptive Statistics}

GDP growth rates under Democratic administration in the second, third, and the fourth years are greater than growth under a Republican administration. The difference in the second year has enlarged since 1953, while the differences in the third and fourth years have shrunk during the second half of the 20th century. The average cumulative GDP growth for four years under Democratic administration is 18.51 percent since 1900 and 15.33 percent since 1953, while under Republican presidents the numbers are 10.71 percent and 11.21 percent, respectively (see Figure 1).

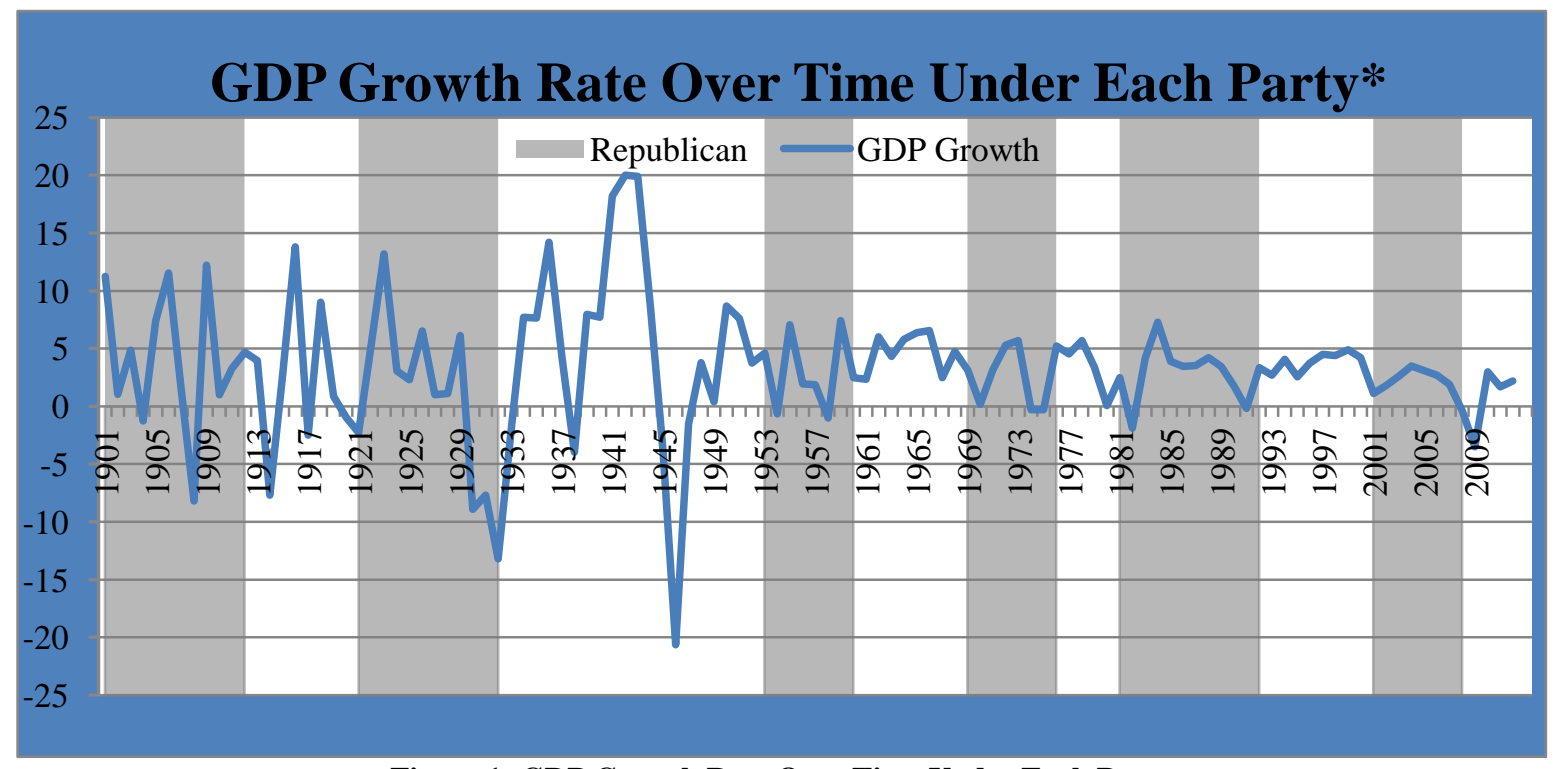

Figure 1: GDP Growth Rate Over Time Under Each Party

* The grey area is Republican administration and the blank area is Democratic administration.

The average Republican administration unemployment rate is higher as compared to Democratic. In addition, Republican presidents tend to govern in an economy with increasing rates, while Democratic presidents govern over declining unemployment rates. The average unemployment rate under a Republican administration increased from 5.21 in the first year to 6.99 in the last year. This compares to the average unemployment rate under a Democratic administration which shows a negative trend. The average unemployment rates of the four years within a Democratic presidency term are 7.12, 6.62, 6.03, and 5.49, respectively (see Figure 2).

Table 3 briefly compares each after-election DJI movement (using Wednesday change as the indicator) and economic performance within a four-year administration. Since in the long run the US GDP growth rate keeps relatively stable (the log US GDP line is famous for that it is almost straight except for some special years), the difference between the GDP growth under each administration and the average GDP growth since 1900 were used as the indicator of economic growth. The researchers also use the difference between the average unemployment rate under each administration and the average unemployment rate since 1900 as the other indicator of economy. If the DJI moves positively, the assumption was that the market is optimistic about the future economy and expects a higher growth rate and lower unemployment rate, and vice versa. If the future economic indicators met the market expectation, the researchers assess the market conclusion as correct. 


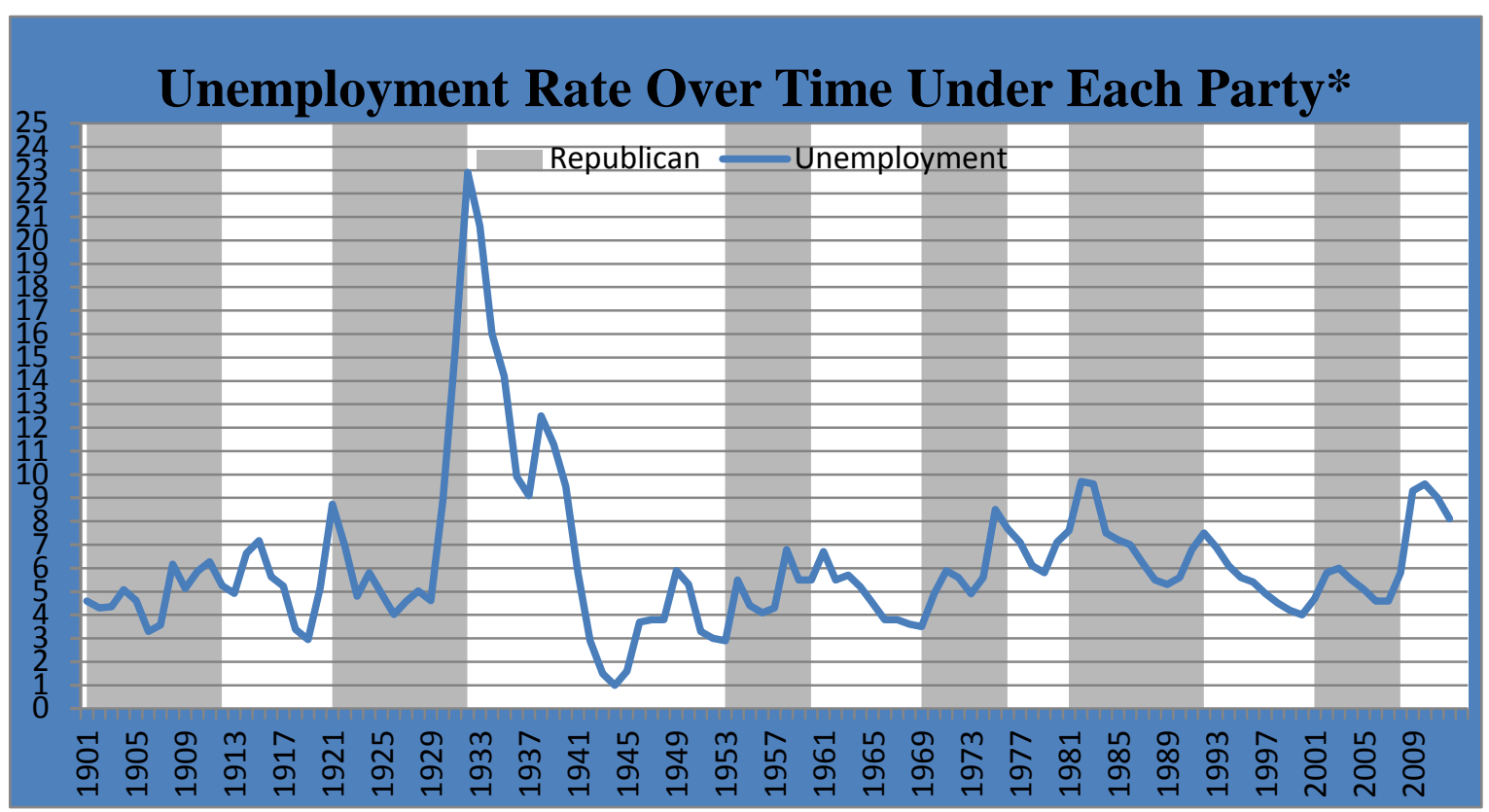

Figure 2: Unemployment Rate Over Time Under Each Party

* The grey area is Republican administration and the blank area is Democratic administration.

The data of the individual 28 administrations are divided into three groups with equal length of years. From Table 3, it is clear that the market has been more and more accurate in predicting the GDP growth, especially after 1972. From McKinley to the end of Roosevelt's first term, the market was only three times right about the GDP growth and seven times right about the unemployment rate level. From 1936 to the end of Nixon's second term, the market was four times right about the GDP growth and three times right about the unemployment rate level. From 1972 to the end of Bush's second term, the market was seven times right about the GDP growth and four times right about the unemployment rate level. If the increasing accuracy of prediction future GDP growth implies the market's greater ability of integrating the candidate's information, it seems that the market's movement after election did not reflect investors' expectation of the unemployment rate. Instead of concluding that the market is not good at predicting the future unemployment rate under a specific administration, an alternative explanation is that compared to economic growth, the unemployment rate is relatively less important in determining the market's movement direction, under the assumption that the market is more and more efficient in evaluating a presidential candidate and his party.

Figures 3, 4, and 5 show more information by presenting the evolution of historical correlations between DJI change after Election Day and other indicators of interest. Figure 3 shows the evolution of correlation coefficients between DJI change after Election Day and GDP growth. The calculation of correlation coefficients in Figure 3 is as follows. For each observation, the value is the correlation coefficient of two time series data, each containing 10 numbers which indicate 10 administrations in past years - one is the DJI 1-Day Change from Table 1 and the other is the Cumulative GDP Growth from Table 2. For example, the value of the observation in 1940 (equal to -0.52) is the correlation coefficient of DJI 1-Day Change from 1904 to 1940 (for observation in 1972, it is from 1936 to 1972, across 40 years and 10 administrations) and Cumulative GDP Growth during the same period. The higher value in later years means the greater match between DJI change after Election Day and the cumulative GDP growth under the president's four-year administration.

The other figures are generated in a similar way. Figure 4 presents the evolution of correlation coefficients between DJI changes after Election Day and the average unemployment rate, and Figure 5 presents the evolution of correlation coefficients between DJI change after Election Day and the overall DJI return within four years. 


\section{Evolution of Correlation between DJI Change after Election Day and GDP Growth of Past 10 Administrations}

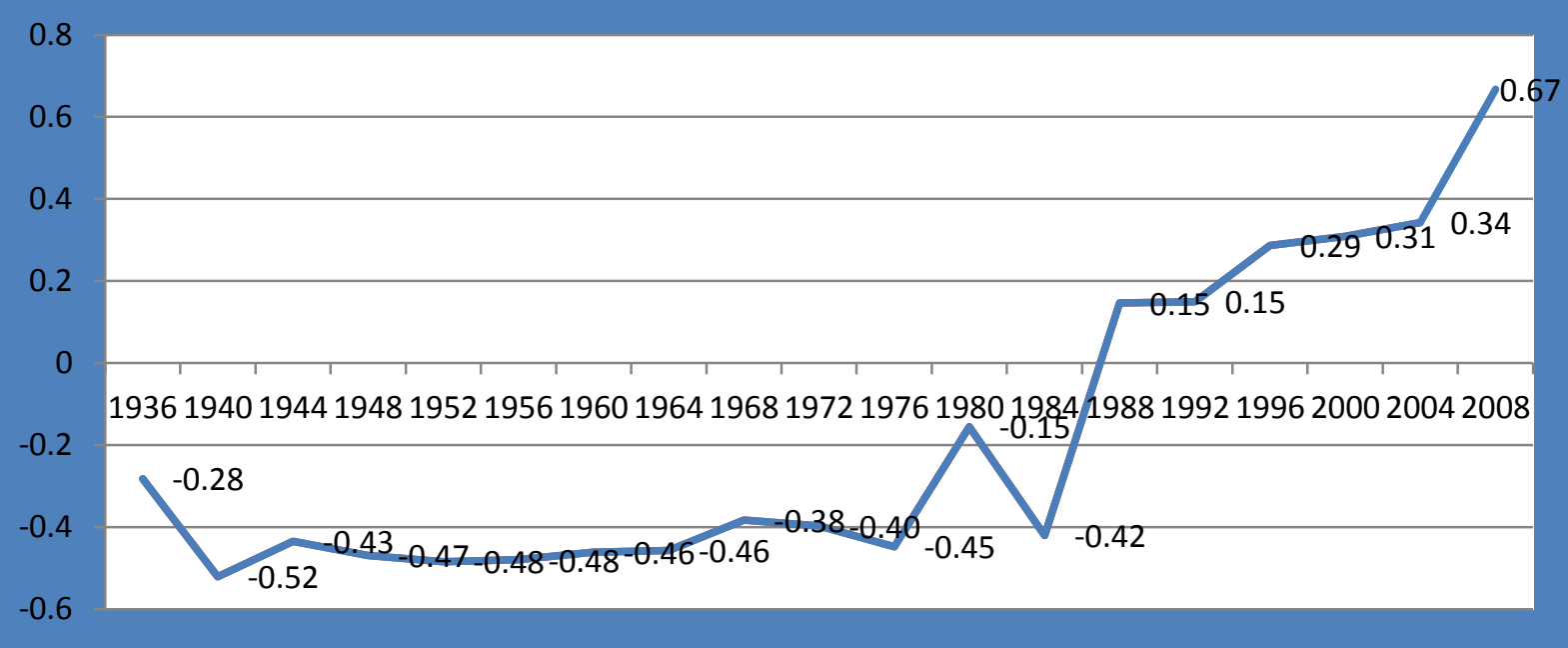

Figure 3: Evolution of Correlation between DJI Change after Election Day and GDP Growth of Past 10 Administrations

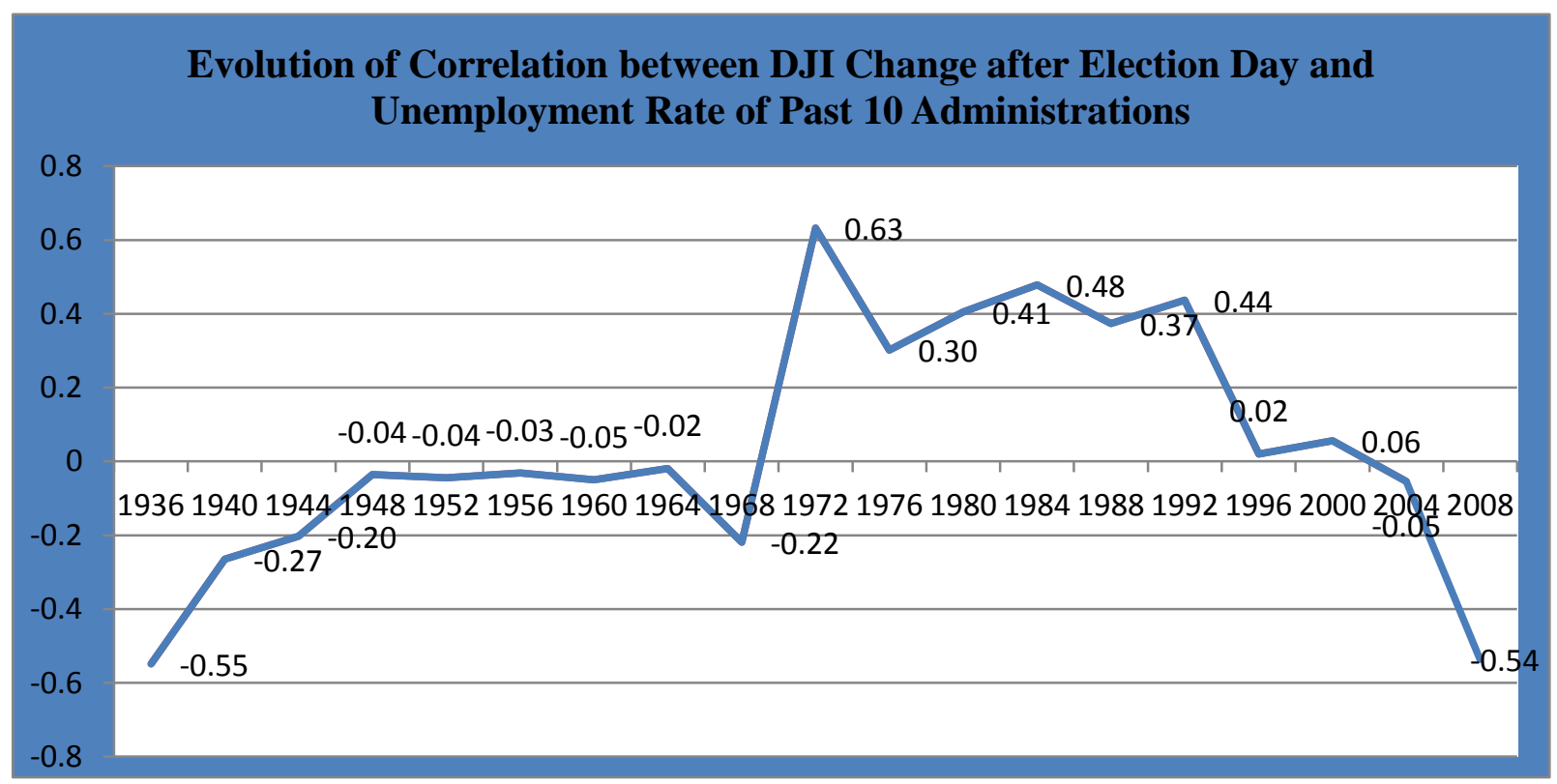

Figure 4: Evolution of Correlation between DJI Change after Election Day and Unemployment Rate of Past 10 Administrations

The figures show that the market has been more and more accurate and efficient in predicting the future GDP growth and the future market return under a president, as the correlation coefficients are increasing. However, the correlation coefficients between the DJI change after election and the average unemployment rate keep around zero constantly for most of the time. Although the numbers decline in Figure 5 and 4 very recently, the trend is not as clear as in the 4-year GDP growth and DJI return figures. 


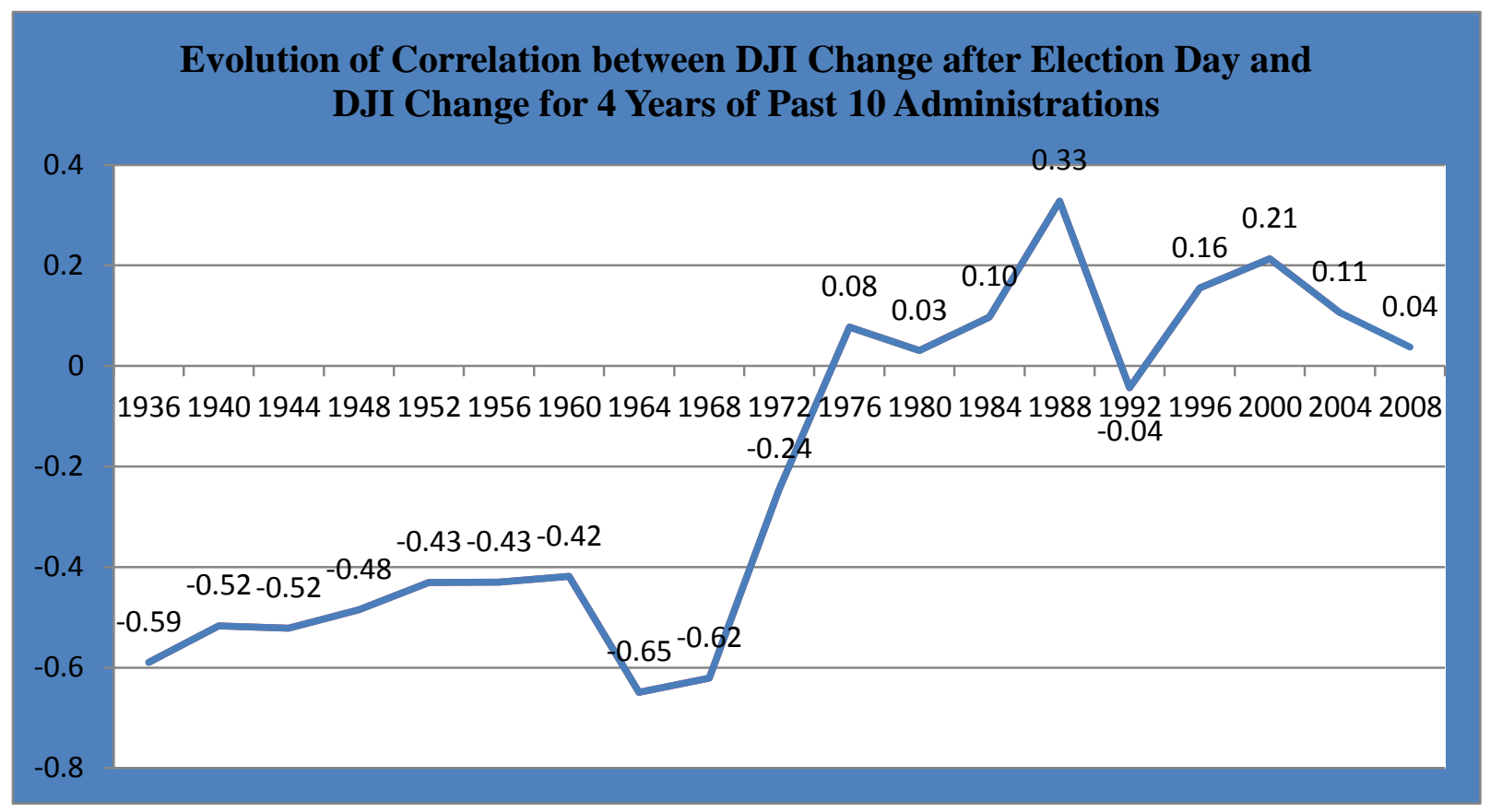

Figure 5: Evolution of Correlation between DJI Change after Election Day and DJI Change for 4 Years of Past 10 Administrations

There is a possibility that the results are due to coincidence since the correlation analysis does not ensure the causality, as most research has encountered similar problems; but each of stock market, economic indicators, and presidential election attracts people's attention and it is useful to show readers the important relationships between them.

\section{RESULTS}

Table 4 shows six regressions of which (1), (2), and (5) show the results of a sub-sample of the most recent ten administrations from Richard Nixon/Gerald Ford in 1972. Models (1) and (2) show that for the most recent ten administrations, the 1-day DJI percentage change following the Election Day is a significant predictor of the cumulative GDP growth of the following four years. However, Models (3) and (4) show that the prediction has no significant accuracy when all 28 administrations since 1900 are included. Models (5) and (6) show that the 1-day DJI percentage change following the Election Day and the average unemployment rate of the following four years has no correlation in both the sub-sample and the full sample.

Table 4: Results of Regressions

\begin{tabular}{|c|c|c|c|c|c|c|}
\hline Model & (1) & (2) & (3) & (4) & (5) & (6) \\
\hline & $\begin{array}{c}\text { Cumulative } \\
\text { GDP } \\
\text { Growth } \\
\end{array}$ & $\begin{array}{c}\text { Cumulative } \\
\text { GDP } \\
\text { Growth }\end{array}$ & $\begin{array}{c}\text { Cumulative } \\
\text { GDP } \\
\text { Growth }\end{array}$ & $\begin{array}{c}\text { Cumulative } \\
\text { GDP } \\
\text { Growth } \\
\end{array}$ & $\begin{array}{c}\text { Average } \\
\text { Unemployment }\end{array}$ & $\begin{array}{c}\text { Average } \\
\text { Unemployment }\end{array}$ \\
\hline DJI 1-Day Change & $\begin{array}{l}1.660^{*} \\
(0.699)\end{array}$ & $\begin{array}{l}1.533^{*} \\
(0.605)\end{array}$ & $\begin{array}{l}-0.677 \\
(1.798)\end{array}$ & $\begin{array}{l}-1.918 \\
(1.678)\end{array}$ & $\begin{array}{l}-0.385 \\
(0.214)\end{array}$ & $\begin{array}{l}-0.294 \\
(0.266)\end{array}$ \\
\hline $\begin{array}{l}\text { Lag Cumulative } \\
\text { GDP Growth }\end{array}$ & $\begin{array}{r}-0.190 \\
(0.431) \\
\end{array}$ & & $\begin{array}{l}-0.334 \\
(0.205)\end{array}$ & & & \\
\hline Constant & $\begin{array}{l}14.35^{*} \\
(5.528)\end{array}$ & $\begin{array}{l}11.97^{* * * *} \\
(1.166)\end{array}$ & $\begin{array}{l}19.34^{* * * *} \\
(4.496)\end{array}$ & $\begin{array}{l}14.25^{* * * *} \\
(3.325)\end{array}$ & $\begin{array}{l}6.327^{* * * *} \\
(0.412)\end{array}$ & $\begin{array}{l}6.206^{* * * *} \\
(0.528)\end{array}$ \\
\hline$N$ & 10 & 10 & 28 & 28 & 10 & 28 \\
\hline$R^{2}$ & 0.460 & 0.446 & 0.139 & 0.048 & 0.288 & 0.045 \\
\hline
\end{tabular}

Standard Errors In Parentheses, ${ }^{*} \mathrm{p}<0.05,{ }^{* *} \mathrm{p}<0.01,{ }^{* * *} \mathrm{p}<0.001$ 


\section{CONCLUSION}

The authors' study adds to the literature on examining the relationship between a presidential administration and the economy. The researchers demonstrated that GDP growth is associated with the prediction of the Wall Street, as defined by the change in stock price immediately after the election. This relationship has strengthened over time. The researchers were not able to identify the same relationship between stock market change immediately after an election and unemployment. The divergent results may be explained by the political economy framework and the strong relationship between business and the presidential administration. Given that the focus of business is on growth and not full employment, these results suggest that when Wall Street casts its prediction after an election, the prediction focuses on growth and excludes unemployment variables. Additional research is needed to determine how GDP, unemployment, and presidential policies interrelate.

\section{AUTHOR INFORMATION}

Wen-Wen Chien is an Assistant Professor of Accounting at State University of New York at Old Westbury. E-mail: chienw@oldwestbury.edu (Corresponding author)

Roger W. Mayer is an Instructor at Walden University. E-mail: roger.mayer@ waldenu.edu

Zigan Wang is a PhD candidate in economics at Columbia University. E-mail: zw2160@ columbia.edu

\section{REFERENCES}

1. Allvine, F. C., \& O’Neil, D. E. (1980). Stock market returns and the presidential election cycle: Implications for market efficiency. Financial Analysts Journal, 36, 49-56.

2. Barro, R. J. (1977). Unanticipated money growth and unemployment in the United States. The American Economic Review, 67, 101-115.

3. Caro, R. (2002). The years of Lyndon Johnson: Master of the Senate. New York, NY: Alfred Knopf.

4. Darby, M. R. (1976). Three-and-a-half million U.S. Employees have been mislaid: Or, an explanation of unemployment. Journal of Political Economy, 84, 1934-1941.

5. Fama, E. (1970). Efficient capital markets: A review of theory and empirical work. Journal of Finance, 25, 383-417.

6. Gilpin, R. (2001). Global political economy: Understanding the international economic order. Princeton, NJ: Princeton University Press.

7. Goodell, J. W., \& Bodey, R. A. (2012). Price-earnings changes during US presidential election cycles: Voter uncertainty and other determinants. Public Choice, 150, 633-650.

8. Goodell, J. W., \& Vähämaa, S. (2013). U.S. presidential elections and implied volatility: The role of political uncertainty. Journal of Banking \& Finance, 37, 1108-1117. Retrieved from http://dx.doi.org/10.1016/j.jbankfin.2012.12.001

9. Huang, R. D. (1985). Common stock returns and presidential elections. Financial Analysts Journal, 41, 58-61.

10. Johnson, R. R., Chittenden, W. T., \& Jensen, G. R. (1999). Presidential politics, stocks, bonds, bills, and inflation. The Journal of Portfolio Management, 26, 27-31.

11. Jones, S. T., \& Banning, K. (2009). U.S. elections and monthly returns. Journal of Economics and Finance, 33, 273-287.

12. Maddison, A. (2008). Historical statistics of the world economy: 1-2008 Ad, dataset. Retrieved from http://rwanda.opendataforafrica.org/xpjarsb

13. Niederhoffer, V., Gibbs, S., \& Bullock, J. (1970). Presidential elections and the stock market. Financial Analysts Journal, 26, 111-113.

14. Riley, W. B., \& Luksetich, W.A. (1980). The market prefers Republicans: Myth or reality. Journal of Financial and Quantitative Analysis, 15, 541-560.

15. Romer, C. (1986). Spurious volatility in historical unemployment data. The Journal of Political Economy, 94, 1-37. 
16. Santa-Clara, P., \& Valkanov R. (2003). The presidential puzzle: Political cycles and the stock market. The Journal of Finance, 58, 1841-1872.

17. U.S. Bureau of Labor Statistics. (2013). Labor force statistics from the current population survey. Retrieved from http://data.bls.gov/pdq/SurveyOutputServlet

18. Wisniewski, T. P., Lightfoot, G., \& Lilley, S. (2012). Speculating on presidential success: Exploring the link between the price-earnings ratio and approval ratings. Journal of Economics and Finance, 36(1), 106-122. 